physically realised ... by quasi-equivalent bonding of identical units.'

Support for the quasi-equivalent bonding scheme comes from the oocyte cables. Optical diffraction shows that the filaments repeat after 19 turns of the actin helix containing 41 subunits. The model predicts that 9 of the 41 subunits are appropriately oriented for quasi-equivalent bonding, and the cables reveal nine transverse stripes at the predicted axial positions in each repeat. These are thought to be due to the 55,000 molecular weight protein attaching to and crosslinking actin subunits of adjacent filaments at these points.

In the case of the acrosomal process no transverse stripes are seen. But here every actin is associated with the 55,000 component, not just those involved in crosslinking, so that all morphological subunits look alike. A bonding scheme analogous to that in the oocyte bundles has been proposed, with only those 55,000 components attached to appropriately oriented actins actually participating in crosslinking. The model seems plausible since the bond distortions in this case are less than those required in the oocyte bundles. DeRosier et al. have carried out a three-dimensional reconstruction of the acrosomal bundle. This reveals that the 55,000 molecular weight protein is bound to the actin subunit near the myosin binding site. While this may not be significant in a cell thought not to have myosin, it could prove relevant in other systems in controlling the interaction of actin cables with myosin.

This is the first time that actin filament cables from cells have been subjected to such analysis. The results, showing how the 55,000 molecular weight protein binds the filaments into an organised cluster, may be applicable to bundles from other sources-but with caution, since different or additional controls may prevail elsewhere.

\section{Free calcium measurement in cells}

\author{
from T. J. Lea
}

An EMBO Workshop on $\mathrm{Ca}$ Measurement in Cells was held in Oxford on 8-10 July, 1977.

IT was in 1928 that $H$. Pollack reported that a saturated solution of alizarin sulphonate when injected into amoebae produced a shower of red crystals ( $\mathrm{Ca}$ alizarinate) in the cyto- plasm on the initiation of pseudopod formation. This pioneering attempt to examine cellular changes in free $\mathrm{Ca}$ was mentioned at the workshop during discussions of more modern methods. In a three-cornered fight between the users of photoproteins (such as aequorin), metallo-chromic indicators (such as arsenazo III) and Ca-sensitive microelectrodes, limitations of these more recently developed methods were critically examined and it became apparent that no single technique satisfied all the requirements of a good indicator of free $\mathrm{Ca}$ in cells.

On the evidence of results achieved, aequorin has until now led the field. Among recent advances obtained with this photoprotein was the demonstration by C. C. Ashley (University of Oxford) and colleagues of a transmitter-like action of L-glutamic acid on barnacle muscle fibres, and $\mathrm{Ca}$ transients in cardiac muscle (D. G. Allen, University College London). The related indicator obelin has been used in sarcoplasmic reticulum vesicle by D. W. Yates (University of Bristol) and A. K. Campbell (Welsh National School of Medicine, Cardiff) in an attempt to measure $\mathrm{Ca}$ uptake by the vesicles. This method does suffer from the disadvantage of a fairly rapid consumption of the photoprotein by $\mathrm{Ca}$ entering the vesicles. An undoubted highlight of the meeting was the presentation by L. Jaffe (Purdue University, Indiana) on aequorininjected eggs of the medaka, a freshwater fish. Jaffe and his colleagues succeeded in showing that immediately following fertilisation, a Ca-wave crosses a single egg (1 $\mathrm{mm}$ diameter) from the point of entry of the sperm and reaches the opposite side within two minutes. He concluded that because the wave is unaffected by Ca-free media, its mechanism involves a calcium-induced $\mathrm{Ca}$ release from some internal sites, and this idea was certainly of interest to muscle physiologists present in the audience.

The problem of quantifying the $\mathrm{Ca}$ measuring methods especially in relation to resting free $\mathrm{Ca}$ in cells was an important theme throughout the meeting. Allen produced evidence of a Ca-independent light emission from aequorin in vitro which he claimed gave a theoretical detection limit for free $\mathrm{Ca}$ in cells of $6 \times 10^{-8} \mathrm{M}$ with a practical limit of $3 \times 10^{-7} \mathrm{M}$. Since A. Scarpa (University of Pennsylvania) and P. F. Baker (King's College, London) estimated the free $\mathrm{Ca}$ in squid axons using aequorin to be $2 \times 10^{-8} \mathrm{M}$ and $6 \times 10^{-8}-15 \times 10^{-8} \mathrm{M}$ respectively, this particular claim aroused some objections. However, in

T. J. Lea is $M R C$ research assistant, University Laboratory of Physiology, Oxford. general, new data given by Allen and Ashley on the shape of the aequorinCa calibration curve and its dependence on cytoplasmic ions, such as $\mathrm{Na}^{+}, \mathrm{K}^{+}$, $\mathrm{H}^{+}$and $\mathrm{Mg}^{2+}$, should improve the accuracy of free $\mathrm{Ca}$ determinations made with this photoprotein.

In the opinion of I. Parker (University College, London), the absorbance indicator arsenazo III is superior to aequorin for measuring rapid transient changes in free $\mathrm{Ca}$, such as those in the sarcoplasm and nerve endplate of a frog muscle fibre during a single twitch. The response time is faster, although how much faster seemed to be disputed, and the free $\mathrm{Ca}$ is linearly related to the absorbance change. For longer term events such as muscle tetani, he felt arsenazo to be inferior because of large movement artefacts, drift in the optical record and unexplained 'tail effects' at the end of the event. L. H. Pinto (Purdue) reported the successful use of purified arsenazo in Limulus photoreceptors, but found that concentrations of the dye greater than $3 \mathrm{mM}$ in the cell caused changes in the membrane response to light pulses, which could be explained by arsenazo buffering of cell $\mathrm{Ca}$. The strong dependence of the arsenazo absorbance on $\mathrm{Mg}^{21}$ and $\mathrm{H}^{+}$was also emphasised by several speakers. The limit of Ca detection by arsenazo was put at $\sim 10^{-7} \mathrm{M}$ by J. D. Owen (University of Utah, Salt Lake City).

Of the three methods, the $\mathrm{Ca}$ selective microelectrode has achieved least in the field so far, but it has much to offer, particularly in the measurement of free $\mathrm{Ca}$ in small cells. The use of new compounds embedded in PVC membranes gives the electrodes a linear response down to $10^{-6} \mathrm{M}$ free $\mathrm{Ca}$, while as low as $10^{-8} \mathrm{M}$ can be detected (Owen). In fact $R$. Meech (University of Cambridge) reported a value for the free $\mathrm{Ca}$ in snail neurones very similar to that of Owen's ( $\sim$ $10^{-7} \mathrm{M}$ corrected for $\mathrm{Mg}^{2+}$ ) without the help of a $\mathrm{Ca}$ microelectrode, using instead the reversal potential of the Ca-dependent $\mathrm{K}$ current. Attempts to measure $\mathrm{Ca}$ changes during a single action potential have not so far been successful (G. Christoffersen, Copenhagen) partly because of the slow response time of these electrodes (1-2s).

\section{Correction}

In the article 'Meteorite research old and new' (News and Views 268, 691; 1977) column 3, paragraph 2, 6 lines from end should read: "Serra de Magé minerals probably did not become closed systems until 4.42 Gyr ago," not "4.52 Gyr ago." 